\title{
THERMAL CONSOLIDATION OF POROUS MEDIUM WITH A RHEOLOGICAL KELVIN-VOIGT SKELETON
}

\author{
MONIKA BARTLEWSKA-URBAN \\ Wrocław University of Technology, Institute of Mining, \\ Wybrzeże Wyspiańskiego 27, 50-370 Wrocław, Poland, e-mail: monika.bartlewska@pwr.wroc.pl
}

\section{TOMASZ STRZELECKI}

Wrocław University of Technology, Institute of Geotechnics and Hydrotechnics, Wybrzeże Wyspiańskiego 27, 50-370 Wrocław, Poland, e-mail: tomasz.strzelecki@pwr.wroc.pl

\begin{abstract}
This study presents calculations results of thermal consolidation process of the porous medium with the rheological Kelvin-Voigt skeleton, obtained numerically with the use of Flex.PDE software. The investigated calculation scheme consisted of the porous column filled with a liquid. The vertical load was applied to the top surface of the column through a porous plate allowing the free flow of liquid through this surface. Numerical solution is based on compression of the sample at appropriately defined boundary conditions. The aim of this study was to describe the influence of external load and temperature gradient on the deformation tests progress at different values of three parameters: $\lambda, r^{s}$ and $c_{v}$. The results obtained, in the context of further research, can also be used for the determination of the influence of other parameters of the state and model parameters on the process of thermo poroelasticity of Biot model with rheological skeleton.
\end{abstract}

\section{INTRODUCTION}

The influence of temperature gradient on the process of deformation of porous medium can be called as the thermal process of consolidation. The basic assumptions of the soil and rock mechanics were defined by Kisiel [8] as:

- Assumption of continuity of soil or rock medium, establishment of a multibuilding continuum.

Mathematical model of the porous medium creeping defined as a multicomponent, two-phase body was introduced for the first time by Maurice Biot in 1955-1956 [2], [3]. This model has been analyzed, in a very general way, based on the theory of asymptotic homogenization by method of periodic structures, where the slightly compressible fluid fills the pores of two-phase medium, by Auriault [1], Bensoussan, Lions and Papanicolau [10], Sanchez-Palencia [22], and with statistical methods by Kröner [19], Rubinstein and Torquato [21]. The mathematical model for the case where the gas fills pores of the medium, based on asymptotic homogenization method was presented by Auriault, Strzelecki, Bauer and He [3].

One of the fundamental principles of porous media mechanics is a postulate of the continuity of the porous medium. These models are used for describing the complex 
mediums processes with discontinuous skeleton consisting of soil particles of different size and shape. Models describing the creep processes of this type of soils accurately reflect the real process of deformation of cohesive soils. Experimental studies indicate the important role of layers of water bound by electric filed forces. The presence of bound water causes that the skeleton particles interact with each other through the water in a wide temperature range. The viscosity of the soil skeleton in the creep process of this type of soils plays an important role, in addition to the volume and shape compressibility. This issue was described by Bartlewska [5] in her dissertation as well as by Bartlewska and Strzelecki in [6], [7], [27]. The effect of temperature field in the case of adiabatic processes in the deformation process of two-phase medium composed of elastic skeleton and weakly compressible fluid was described by Coussy. Based on the thermodynamics of irreversible processes he developed a mathematical model of thermal consolidation in [11], and Kowalski et al. in [17], [18]. The problem

of adiabatic processes was also discussed by Strzelecki in [24], [25]. The resulting system of equations by Coussy and Strzelecki has the same form. The differences are in the definition of effective parameters of models.

This paper presents a generalization of Biot's equations to non-isothermal processes, taking into account the rheological characteristics of the skeleton. The proposed model is therefore more complex and includes additional elements resulting from the simultaneous consideration of the elastic characteristics of the liquid or gaseous medium filling the pores, viscous characteristics of the soil skeleton and thermal deformability of two-phase medium. The process of the displacement changes for different values of the coefficient of linear expansion and soil temperature gradient was analyzed. A mathematical model of the thermal consolidation has many effective parameters, which require modern laboratory tools and application of complex statistical methods for their determination. The purpose of this paper is to present the behavior of the soil samples under uniaxial compression and the temperature gradient. Therefore, the process of deformation of the sample during axial compression, and then in a fixed period of time exposed to the temperature gradient is presented. The relationships obtained allow the magnitude of the thermal parameters of the medium to be evaluated with the use of standard geotechnical laboratory equipment. This study thus presents the dependence of displacement of the sample along the center axis on the thermal conductivity $\lambda$ and coefficient of linear expansion of the soil skeleton $r^{s}$.

\section{EQUATIONS OF THERMAL CONSOLIDATION}

Mathematical equations of the thermal consolidation process for the Biot body with rheological skeleton have been derived based on the basic laws of Newtonian mechanics of continuous medium and thermodynamics of irreversible processes. The starting point was the initial assumptions of the theory of two-phase medium com- 
posed of elastic-viscous skeleton and a compressible viscous fluid filling the pores of this medium. The list of assumptions is as follows:

- the porous medium called the Biot body consists of a solid phase, i.e., the medium skeleton and the liquid phase filling the pores,

- the skeleton of the porous medium is the rheological body, which has the characteristic of elasticity and viscosity of shape $\eta_{s}$ and volume $\eta_{s}$,

- deformation of the porous skeleton is small, so strain tensor is a linear Cauchy strain tensor,

- volumetric strain is linear with regard to the solid phase and liquid medium; this assumption is not true when the liquid phase is a gas,

- liquid filtering through the pores of the medium is slightly compressible viscous Newtonian liquid, the filtration process is in the laminar range caused by the pressure gradient in the pores and is given by Darcy,

- the process analyzed is any process which is not isothermal, the system is semiclosed (energy exchange is possible and the exchange of matter in the solid phase is not possible),

- medium is homogeneous and isotropic,

- temperature varies in the range from $0{ }^{\circ} \mathrm{C}$ to $100{ }^{\circ} \mathrm{C}$.

The continuity equation of the solid phase medium is in the form

$$
\frac{D^{S} \bar{\rho}_{S}}{D t}+\bar{\rho}_{S} \dot{\varepsilon}=-\left[\bar{\rho}_{w} v_{i}^{r}\right]_{, i},
$$

and the continuity equation of fluid flow through the center of ground skeleton is given by

$$
\frac{D^{r} \bar{\rho}_{w}}{D t}+\bar{\rho}_{w}(\dot{\theta}-\dot{\varepsilon})=-\left[\bar{\rho}_{w} v_{i}^{l}\right]_{, i},
$$

with the material derivative expressed by the formulas

$$
\frac{D^{s}}{D t}=\frac{\partial}{\partial t}+v_{i}^{s} \frac{\partial}{\partial x_{i}}, \quad \frac{D^{r}}{D t}=\frac{\partial}{\partial t}+v_{i}^{r} \frac{\partial}{\partial x_{i}},
$$

where $v_{i}^{s}$ are the velocity vector components of the soil skeleton, and $v_{i}^{r}=v_{i}^{l}-v_{i}^{s}$ are the relative velocity components of fluid relative to the skeleton velocity. $\bar{\rho}_{s}$ and $\bar{\rho}_{w}$ are the skeleton and the fluid density referenced to the volume of the total area. The $\dot{\theta}$ and $\dot{\varepsilon}$ represent the rate of change of liquid and skeletal dilatation. The equations of motion of the solid phase medium for the case of quasi-static process in which we ignore inertial term of an equation, have the form

$$
\sigma_{i j, j}+X_{i}\left(\bar{\rho}_{s}-\bar{\rho}_{w}\right)=-b v_{i}^{r},
$$


and the equations of motion of the liquid phase in the case of laminar motion are expressed in the formula

$$
\sigma_{, i}+X_{i} \bar{\rho}_{w}=b v_{i}^{r}
$$

where $X_{i}=-\delta_{i 3} g$ is the gravitational acceleration in the right-handed coordinate system, $\sigma_{i j}$ components of the stress tensor in the skeleton, referenced to the total crosssectional area, $\sigma=-p^{*} f_{o}$ the diffuse tension in the fluid filling the pores in porous medium, $b$ the coefficient of viscous resistance of fluid flowing through the soil. Constitutive relations of the Biot body with a Kelvin-Voigt rheological skeleton for nonisothermal processes have the form

$$
\left\{\begin{array}{l}
\sigma_{i j}=2 N \varepsilon_{i j}+M \varepsilon \delta_{i j}+2 N T_{a} \dot{\varepsilon}_{i j}+\left(A T_{b}+N T_{a}\right) \dot{\varepsilon} \delta_{i j}+\frac{Q}{R} \sigma \delta_{i j}+P_{1}\left(T-T_{0}\right) \delta_{i j}, \\
\sigma=Q \varepsilon+R Q+d\left(T-T_{0}\right)
\end{array}\right.
$$

where $N$ is the module of shape deformation of the skeleton, $A$-module of volume deformation of the skeleton, $Q$ - factor of influence of volumetric deformation in the liquid on the tension in skeleton or opposite: factor of the influence of volumetric deformation in the skeleton on the tension in liquid, $R$ - module of volume deformations of the liquid filling the pores of the Biot body. The parameter $M$ is expressed by

$$
M=A-\frac{Q^{2}}{R} .
$$

Constant $d$ is given by

$$
d=-\left[3 Q r^{s}+r^{l} R\right]
$$

where $r^{s}$ and $r^{l}$ denote the linear expansion of the skeleton and the volumetric expansion of the liquid, constant $P_{1}$ is calculated by the formula

$$
P=-\frac{T\left(3 K r^{s}+Q r^{l}\right)}{\lambda}
$$

where $\lambda$ is the coefficient of thermal permeability of soil, $T_{a}$ and $T_{b}$ are the parameters of the skeleton expressed by the formulas

$$
T_{a}=\frac{\eta^{s}}{N} \text { and } T_{b}=\frac{\lambda^{s}}{A},
$$

$\eta^{s}, \lambda^{s}$ are the viscosity of shape and volume of soil skeleton, respectively.

The system of equations of the linear theory of thermal consolidation in the movements of the skeleton and the function of stress in the liquid, the filtration flow equation and the heat conduction equation for the Biot body with a rheological Kelvin-Voigt skeleton consists of five differential equations 


$$
\left\{\begin{array}{l}
N \Psi_{k} \nabla^{2} u_{i}+\left(A \Psi_{L}-\frac{Q^{2}}{R}+N \Psi_{k}\right) \varepsilon_{, i}+\frac{H}{R} \sigma_{, i}-\bar{\rho}_{s} g \delta_{i 3}=-P_{1} T_{, i}, \\
\frac{k R}{f_{o}^{2} \bar{\rho}_{w} g} \nabla^{2} \sigma=T_{0}\left[\dot{\sigma}-H \dot{\varepsilon}+P_{4} \dot{T}\right], \\
\lambda \nabla^{2} T=T_{0}\left[P_{2} \dot{\varepsilon}-P_{3} \dot{\sigma}+P_{5} \dot{T}\right],
\end{array}\right.
$$

where $\Psi_{k}=1+T_{a} \frac{\partial}{\partial t}, \Psi_{L}=1+T_{b} \frac{\partial}{\partial t}$ - are differential operators, $k$ is the coefficient of filtration of fluid through a porous medium, $g$ - acceleration due to gravity, and the coefficients $P_{2}, P_{3}, P_{4}$ and $P_{5}$ are given by the equations

$$
\begin{gathered}
P_{2}=3 r^{s}\left(K-\frac{H Q}{R}\right)-R r^{l}, \quad P_{3}=3 r^{s} \frac{Q}{R}+r^{l}, \quad P_{4}=R P_{3}, \\
P_{5}=\frac{\left(3 Q r^{s}+r^{l} R\right)^{2}}{R}+\frac{\left(\bar{\rho}_{s}+\bar{\rho}_{w}\right) c_{v}}{T},
\end{gathered}
$$

$c_{v}$ is the specific heat at constant volume. The above system of equations is solved and is the starting point for work issues.

\section{CONSTRUCTION OF NUMERICAL MODEL FOR THERMAL CONSOLIDATION OF ISSUES UNIAXIAL}

Numerical model of thermal consolidation of the Biot body with a rheological Kelvin-Voigt skeleton for the case of axisymmetrical problems was created by taking as a starting point three-dimensional shape of the soil samples. Solving the problem in a 3D space where the horizontal components of displacement vector are equal to zero we obtain the solution of the oedometric sample. The consolidation process of the porous medium is described by the system of equations (4). Calculations were performed with the use of the finite element method. Effective model parameters are constans and do not depend on the state of stress and temperature. The effective parameters adopted for the calculation have the following values:

- Biot's constant:

$$
\begin{aligned}
& N=2.5 * 10^{7} \mathrm{~N} / \mathrm{m}^{2}, \quad A=5.0 * 10^{7} \mathrm{~N} / \mathrm{m}^{2}, \\
& R=1.5 * 10^{7} \mathrm{~N} / \mathrm{m}^{2}, \quad Q=2.7 * 10^{7} \mathrm{~N} / \mathrm{m}^{2},
\end{aligned}
$$

- Darcy filtration coefficient:

$$
k=10^{-6} \mathrm{~m} / \mathrm{s},
$$


- porosity:

$$
f_{0}=0.35,
$$

- specific heat at constant volume:

$$
c_{v}=2000 \div 8000 \frac{\mathrm{J}}{\mathrm{kg} * \mathrm{~K}},
$$

- heat conductivity coefficient $\lambda$ :

$$
\lambda=0.5 \div 1.5 \mathrm{~J} / \mathrm{ms} \mathrm{K},
$$

- coefficient of linear expansion of the skeleton $r^{s}$ :

$$
r^{s}=5 * 10^{-6} \div 5 * 10^{-4}[1 / \mathrm{K}]
$$

- volumetric expansion coefficient of water:

$$
r^{l}=1 * 10^{-7} 1 / \mathrm{K},
$$

- viscosity of the shape and volume of the skeleton:

$$
\eta=2.2 * 10^{6} \mathrm{~Pa} * \mathrm{~s}, \quad \lambda_{s}=1.0 * 10^{7} \mathrm{~Pa} * \mathrm{~s} .
$$

For the calculation, the sample in the shape of a cylinder with the radius $r=0.025 \mathrm{~m}$ and height $h=0.076 \mathrm{~m}$ was used. The behavior of the samples in the time interval from $10^{-4}$ to $10^{8}$ was observed under the load $2 * 10^{6} \mathrm{~N} / \mathrm{m}^{2}$ applied to the top surface of the sample at the initial moment and the temperature gradient between the lower and upper base of the sample of $80^{\circ} \mathrm{C}$ in the time interval from $10^{3}$ to $10^{5} \mathrm{~s}$. The rest of the time it was assumed that the temperature of the upper and lower surfaces of the sample is $20^{\circ} \mathrm{C}$. Boundary conditions adopted for the problem were that all the displacement vector components are equal to zero. On the top surface of the sample horizontal components of displacement vector of skeleton were assumed to be zero. The bottom surface of the sample was defined as impermeable for water while on the top surface the value of the water pressure was equal to atmospheric pressure. It is assumed that the vertical displacements take place without friction to the vertical cylindrical surface. The value of the horizontal and vertical stress is dependent on the consolidation process. The effect of changes of the soil thermal conductivity coefficient $\lambda=0.5 \div 1.5 \mathrm{~J} / \mathrm{ms} \mathrm{K}$, the coefficient of linear expansion of soil $r^{s}=5 * 10^{-6} \div 5 * 10^{-4}[1 / \mathrm{K}]$ and specific heat of soil $c_{v}=2000 \div 8000 \frac{\mathrm{J}}{\mathrm{kg} * \mathrm{~K}}$ on the process of changing of temperature and settlement of the sample in time was analyzed. The study was conducted using the program FlexPDE v.6 [28]. 


\section{RESULTS OF NUMERICAL CALCULATIONS}

\subsection{THE PROCESS OF CONSOLIDATION}

FlexPDE has an automatic generator for the finite element mesh which is modified during the computation in such a way that the accuracy imposed on calculations has to be met at every stage of the calculation. In our case, however, to simplify the tedious calculations, a fixed grid was used by adopting the division into 30 segments for each dimension, resulting in 5592 items. The second variable element in the case of unsteady-state process, such as we have, is a time step $d t$. The program starts with an initial time step $d t=10^{-5} \mathrm{~s}$, and ends at $d t=2.06^{*} 10^{9} \mathrm{~s}$. Figures 1 and 2 present the distribution of the finite element calculations in the initial phase.

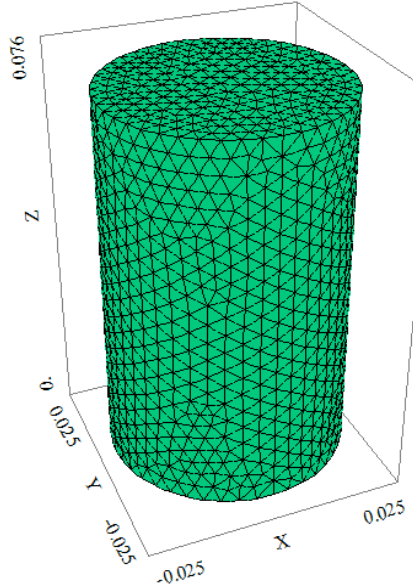

Fig. 1. Finite element mesh generated by the software

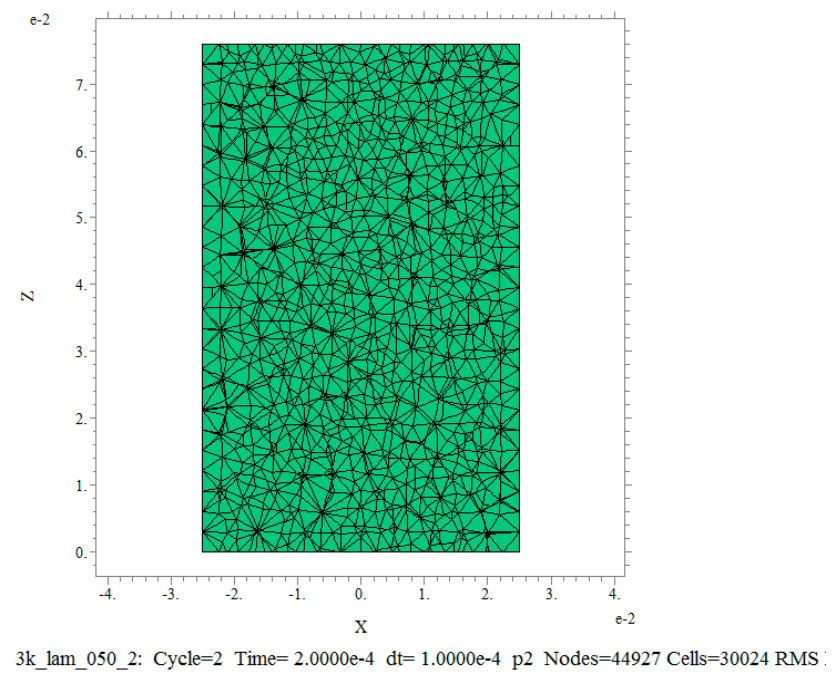

Fig. 2. Initial computational grid vertical axis section

Figures 3 and 4 present displacement vectors for two different simulation times, while Fig. 5 shows a plot of settlement of the sample with logarithmic time scale. As Fig. 5 demonstrates, the value of settlement of the sample under the external load is increasing to the target with time within $t=10 \mathrm{~s}$. The heating at the bottom of the sample starts at $t=10^{3} \mathrm{~s}$ resulting in a temporary reduction of the settlement (change of direction opposite to the force of gravity). At $t=10^{5} \mathrm{~s}$ settlement value returns to the state prior to the heating. This effect is caused by the thermal expansion of porous material. 


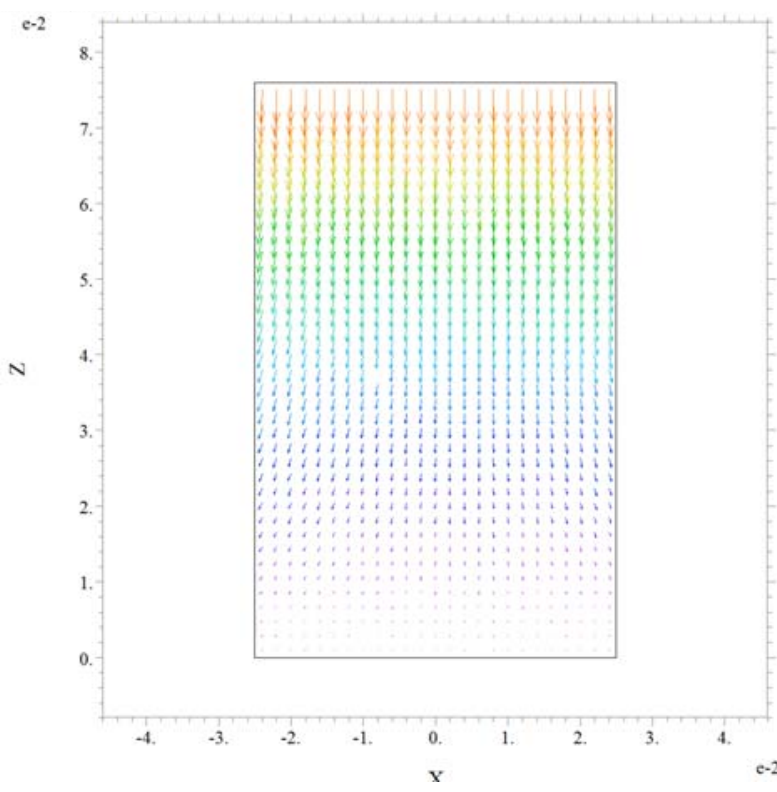

$13: 29: 083 / 12 / 12$

FlexPDE 5,0.7

Fig. 3. Vertical displacement vectors for $t=0.1 \mathrm{~s}$

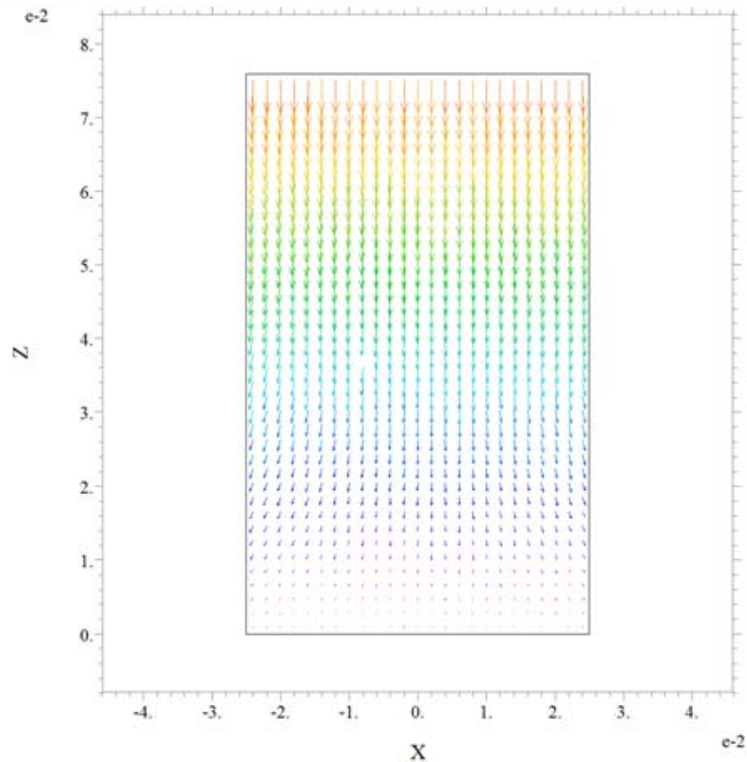

$13: 29: 08$ 3/12/12

FlexPDE 5.0 .7

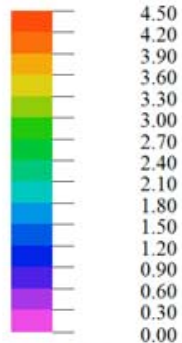

Scale $=E-3$

Fig. 4. Vertical displacement vectors for $t=1 * 10^{8} \mathrm{~s}$ 


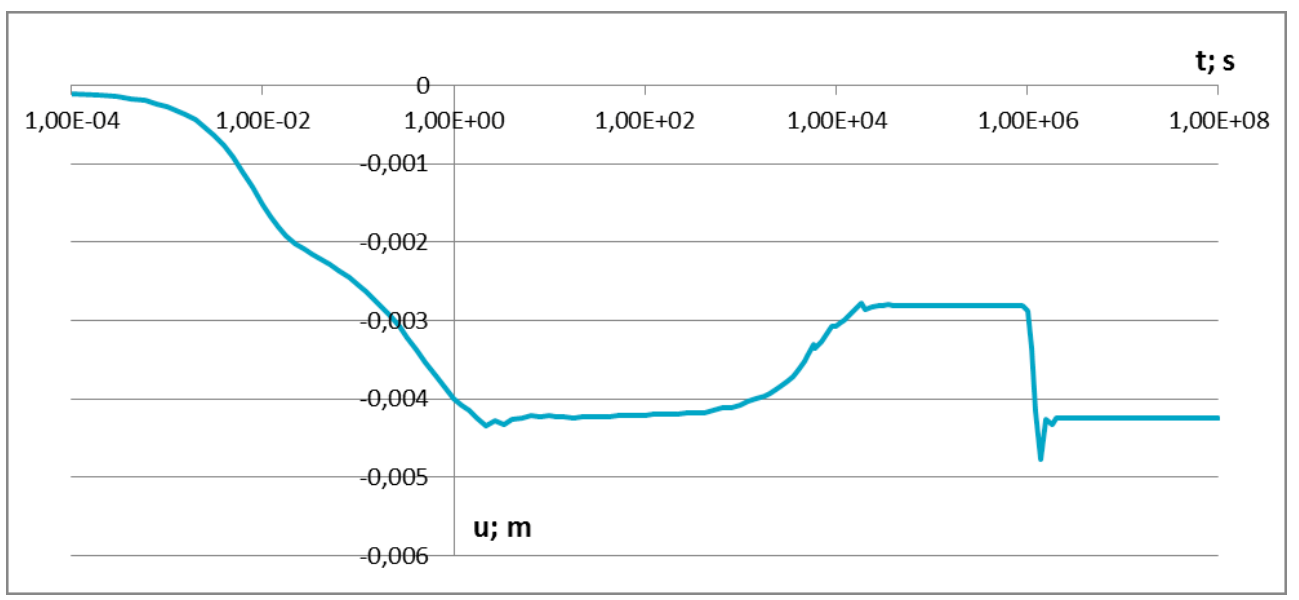

Fig. 5. Diagram of the sample settlements with time

Figures 6 through 11 show the process of temperature change and heat flow during the numerical experiment. In the initial phase of heating the sample, the greatest heat flux values are at the bottom of the sample (Fig. 7), which is caused by an irregular temperature distribution inside the medium (Fig. 6). After reaching the steady state temperature distribution is uniform (Fig. 8) and heat flux at each point has the same value and direction (Fig. 9). At the end of the calculation the temperature is constant for the entire sample (Fig. 10) and heat flow stops (Fig. 11).

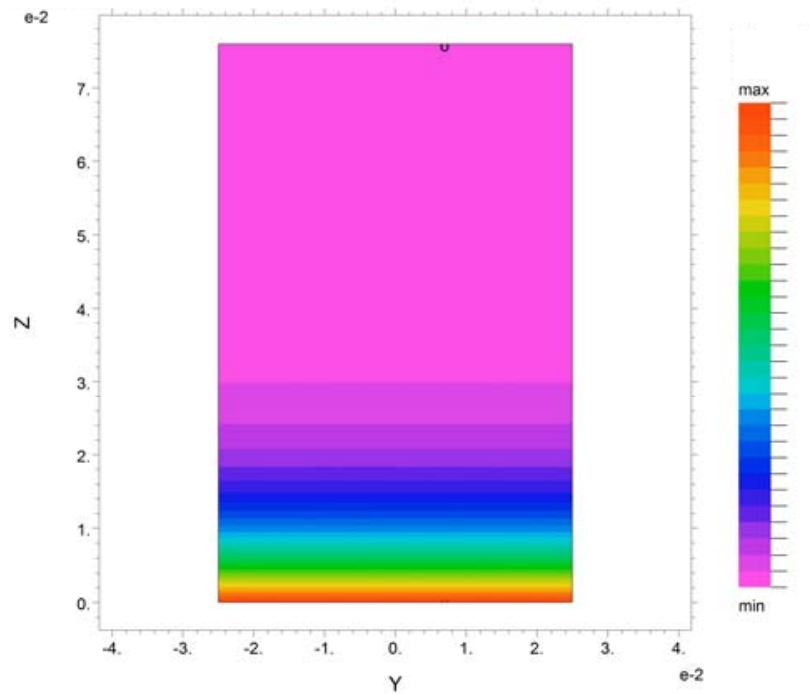

Fig. 6. The temperature distribution for $t=1 * 10^{3} \mathrm{~s}$ 


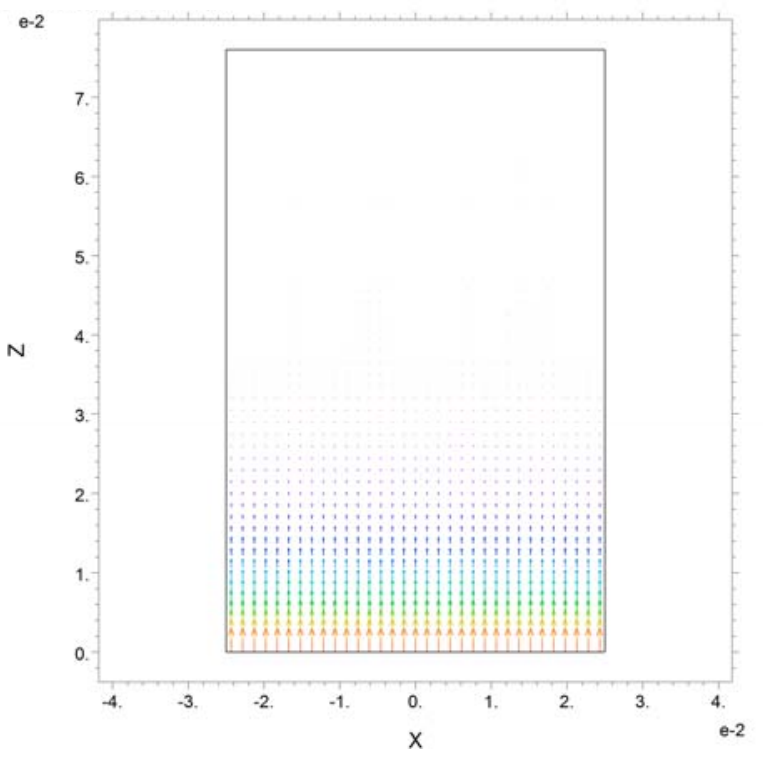

$16: 53: 383 / 17 / 12$

FlexPDE 6.19

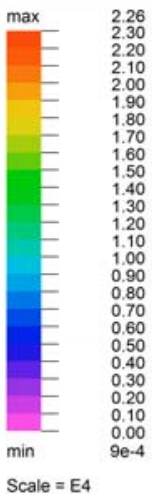

Fig. 7. The field of heat transfer for $t=10^{3} \mathrm{~s}$

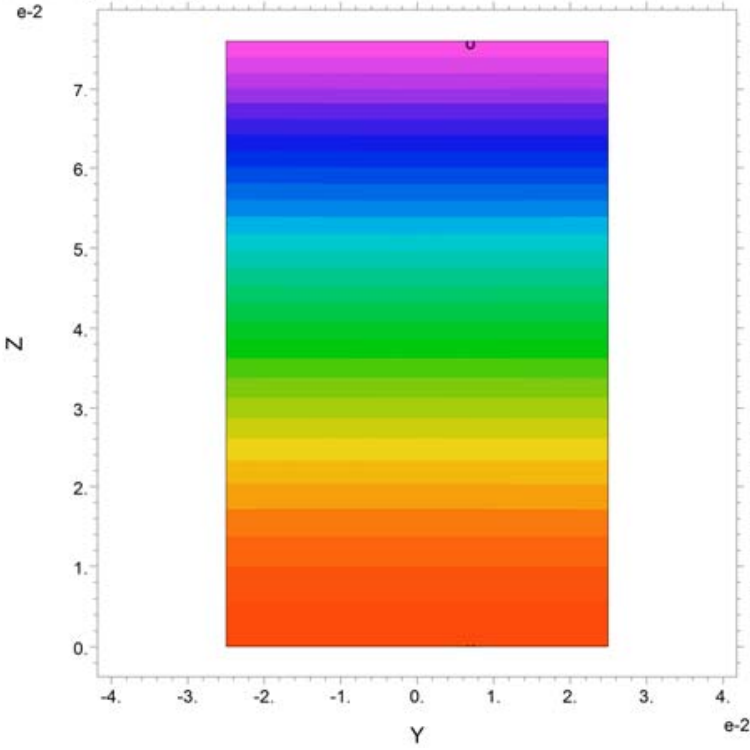

16:53:38 3/17/12
FleXPDE 6.19

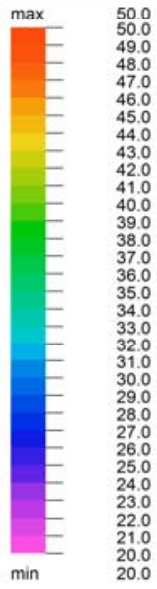

Fig. 8. The temperature distribution for $t=10^{6} \mathrm{~s}$ 


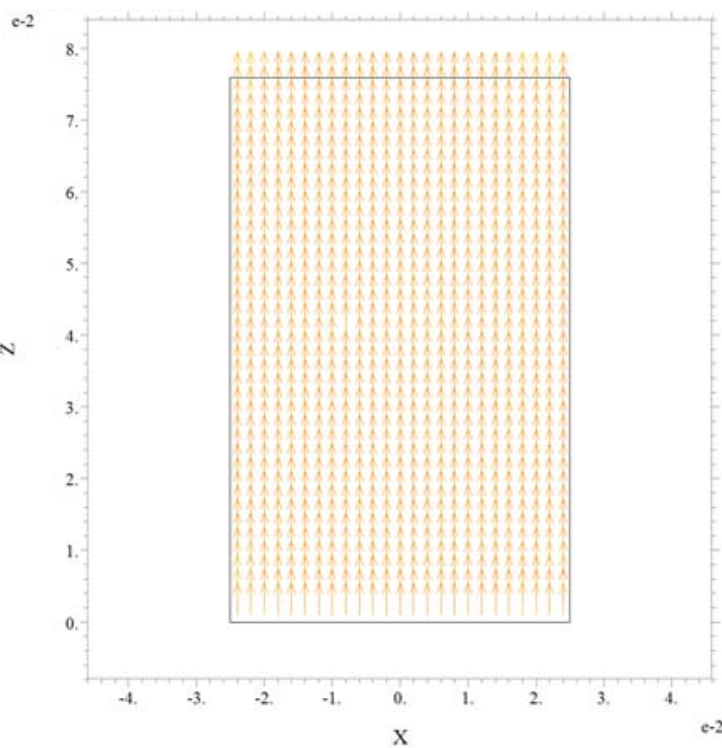

$13: 29: 083 / 12 / 12$

FlexPDE 5.0.7

Fig. 9. The field of heat transfer for $t=9 * 10^{5} \mathrm{~s}$

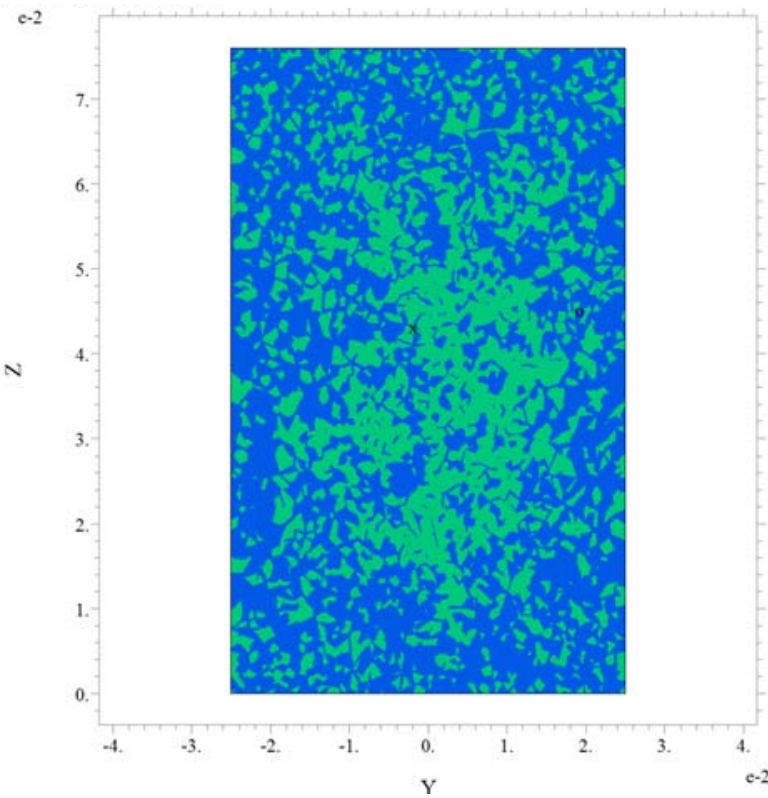

$13: 29: 08 \quad 3 / 12 / 12$

FlexPDE 5.0.7

20.0015

20.0010

20.0005

20.0000

19.9995

19.9990

Fig. 10. The temperature distribution for $t=10^{8} \mathrm{~s}$ 


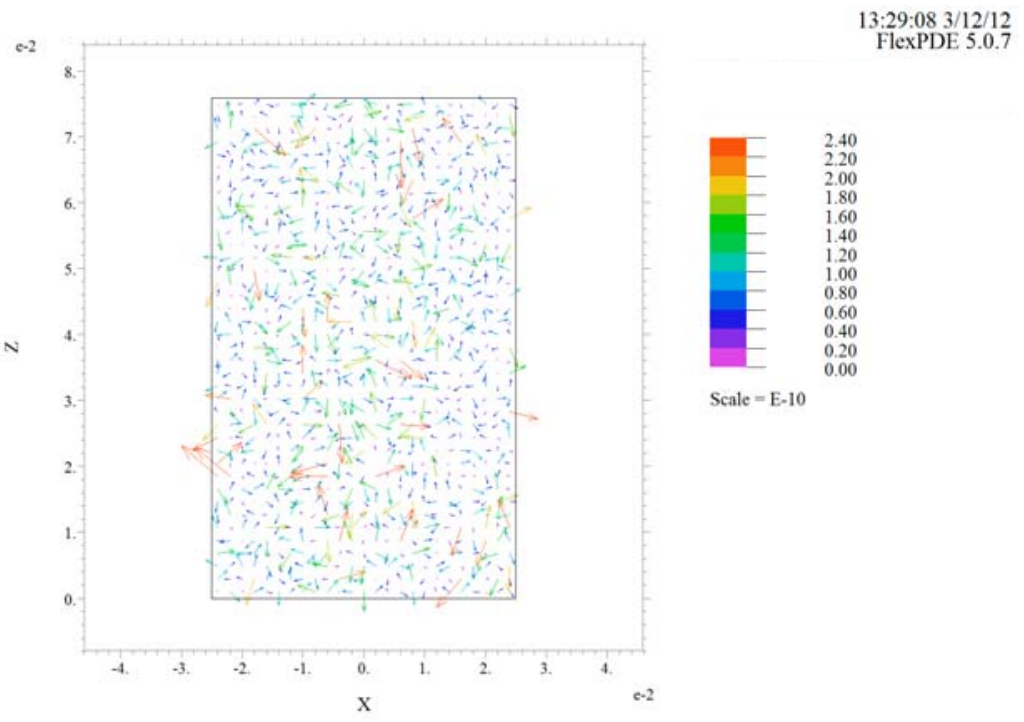

Fig. 11. Heat flow field for the final phase of numerical simulation

The process of filtration of water in the sample by the action of load and temperature fields is illustrated in Figures 12 and 13. Figure 12 shows that the load and temperature field applied to the sample results in nonlinear distribution of the flow velocity field. Figure 13 shows a graph of filtration rate for the time $t=10^{9} \mathrm{~s}$ when the sample is only under the external load. As can be seen from the figure, the temperature field has an influence on the process of filtration of water through the ground.

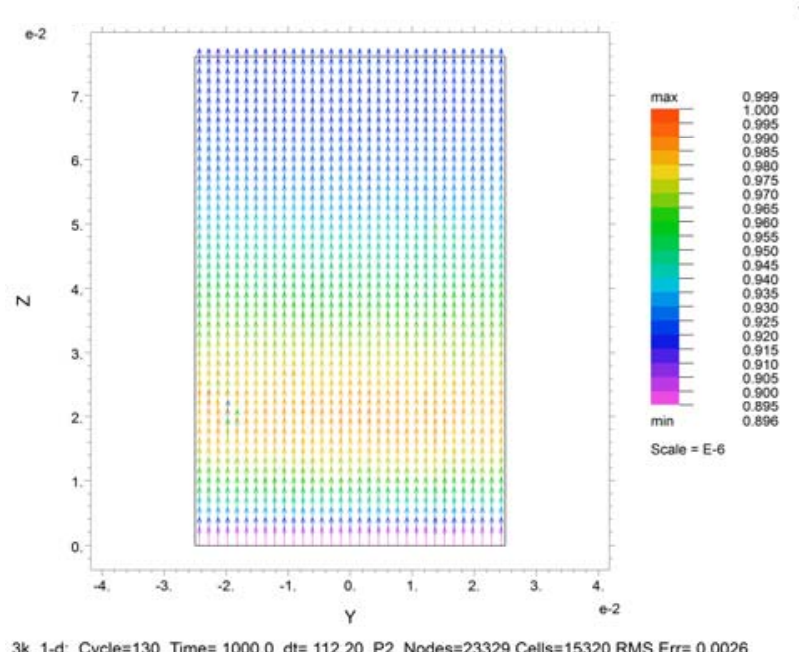

$16: 53: 383 / 17 / 12$
FlexPDE 6.19

Fig. 12. Filtration flow field for the time $t=10^{3} \mathrm{~s}$ 


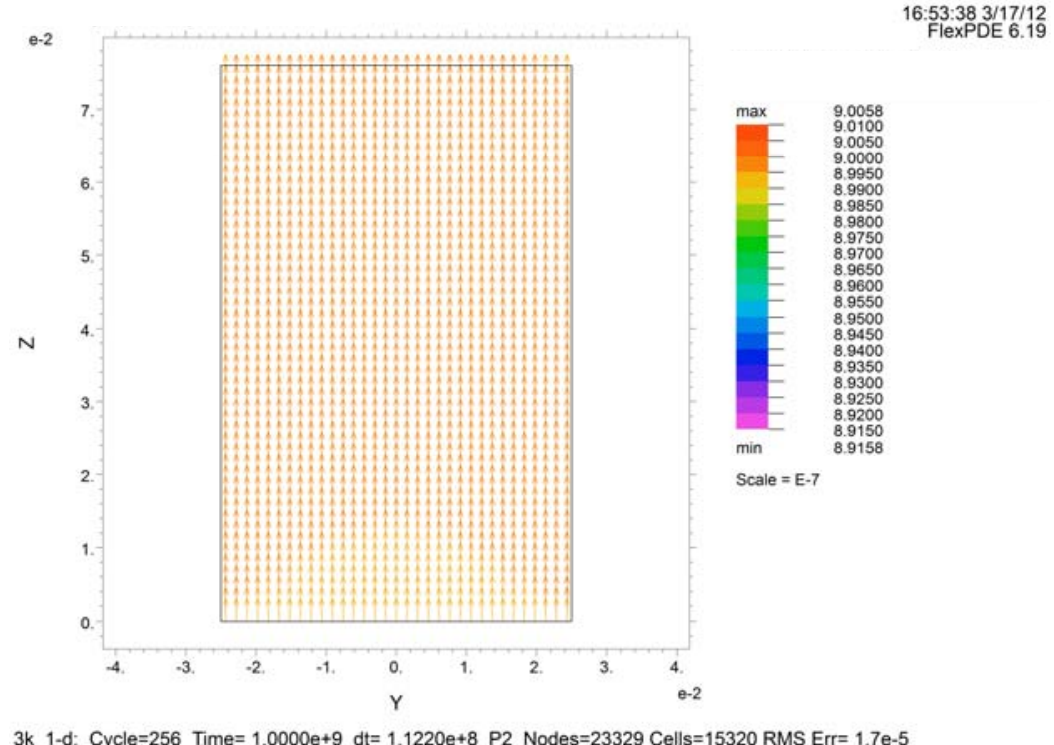

Fig. 13. Filtration flow field for the time $t=10^{9} \mathrm{~s}$

\subsection{INFLUENCE OF SELECTED PARAMETERS ON THE EXPERIMENT}

Changes in the soil thermal conductivity and heat capacity cause the temperature inside the sample to change at varying rate (Figs. 14 and 15) and consequently the rate of sample volume change during heating (Figs. 16 and 17), but these do not affect its

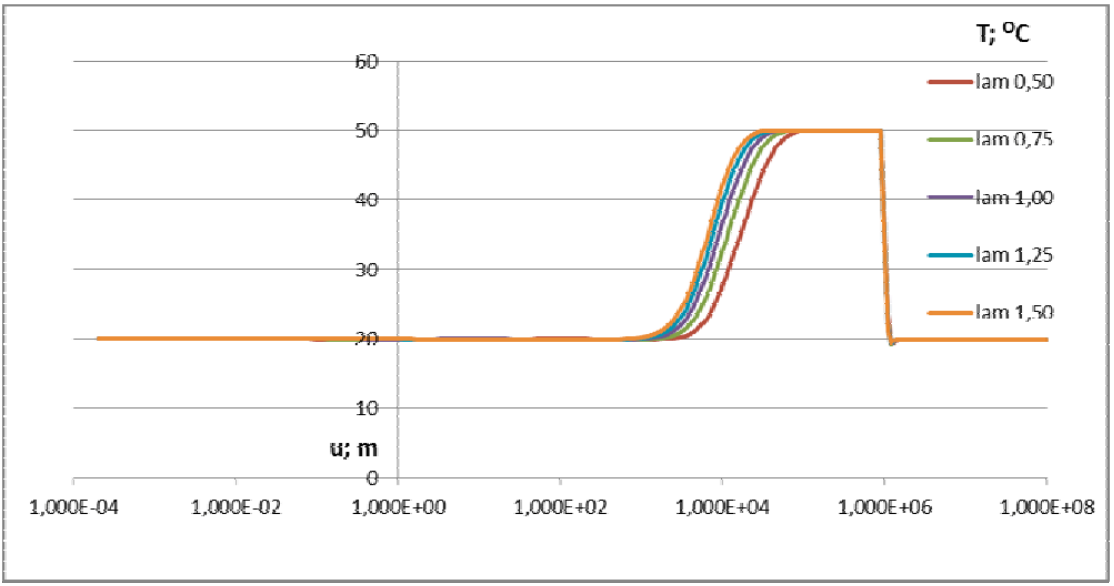

Fig. 14. The plot of temperatures in the center of the sample in time for different values of the coefficient of thermal conductivity 
shape for the ending time of the numerical experiment. The higher the value of the thermal conductivity and the lower the specific heat of soil medium, the faster the process temperature changes and the steady state occurrs. In the case of the coefficient of thermal conductivity of soil, the differences in the rate of temperature rise are caused by the change of heat flux that enters the interior of the sample (Figs. 18 and 19). In the case of the specific heat coefficient changes the flows remain unchanged while the greater part of the flow is stopped inside the sample.

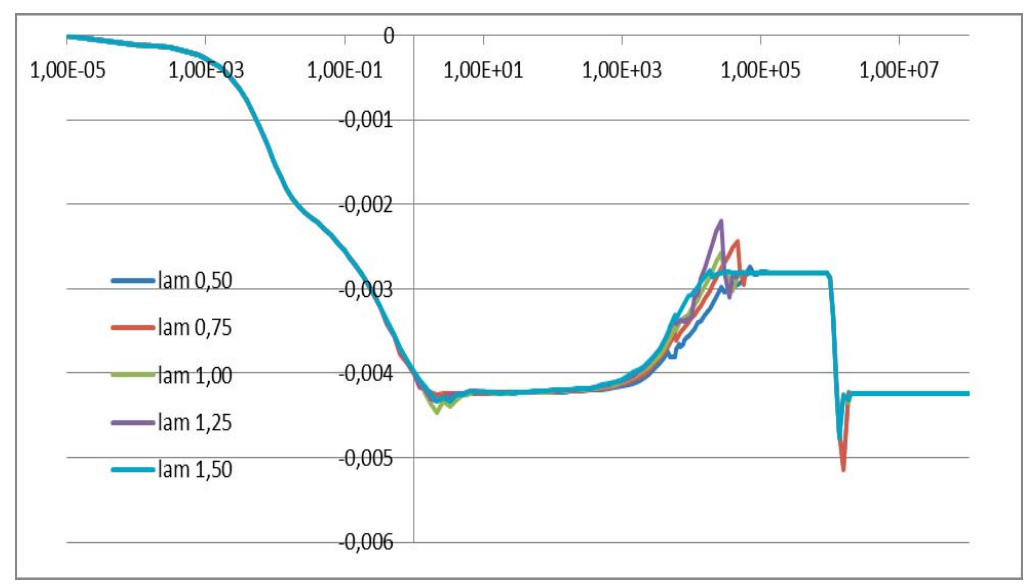

Fig. 15. Displacements of the upper surface of the sample in time for different values of the coefficient of thermal conductivity

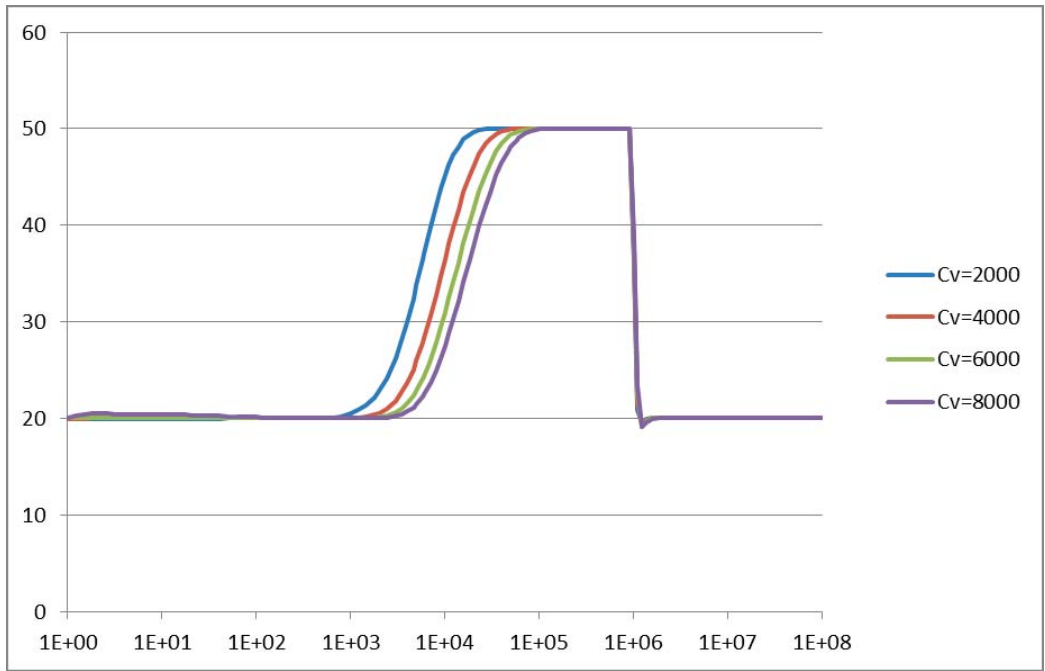

Fig. 16. The plot of temperatures in the center of the sample in time for different values of heat of the ground 


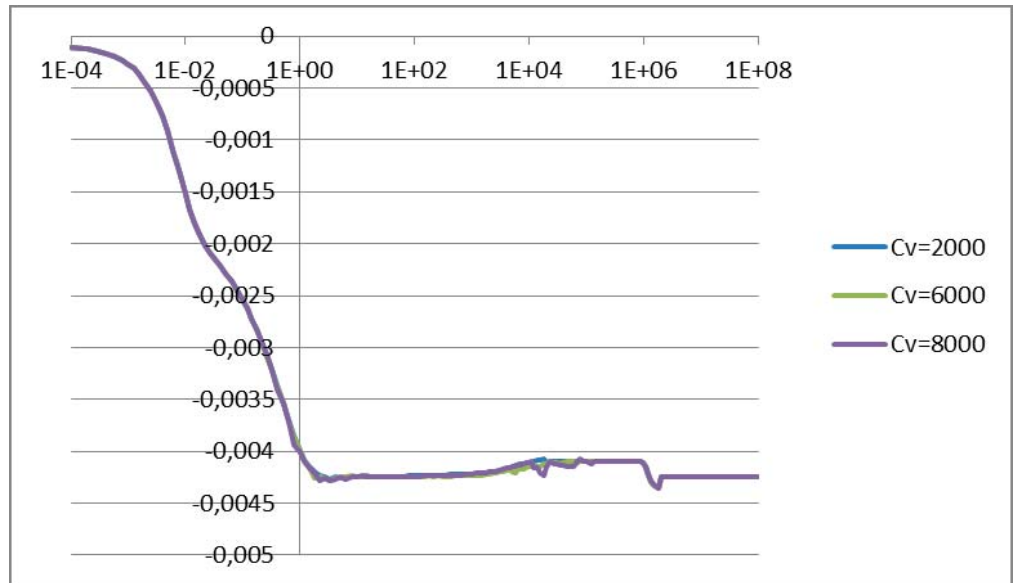

Fig. 17. Displacement of the upper surface of the sample in time for different values of heat of the ground

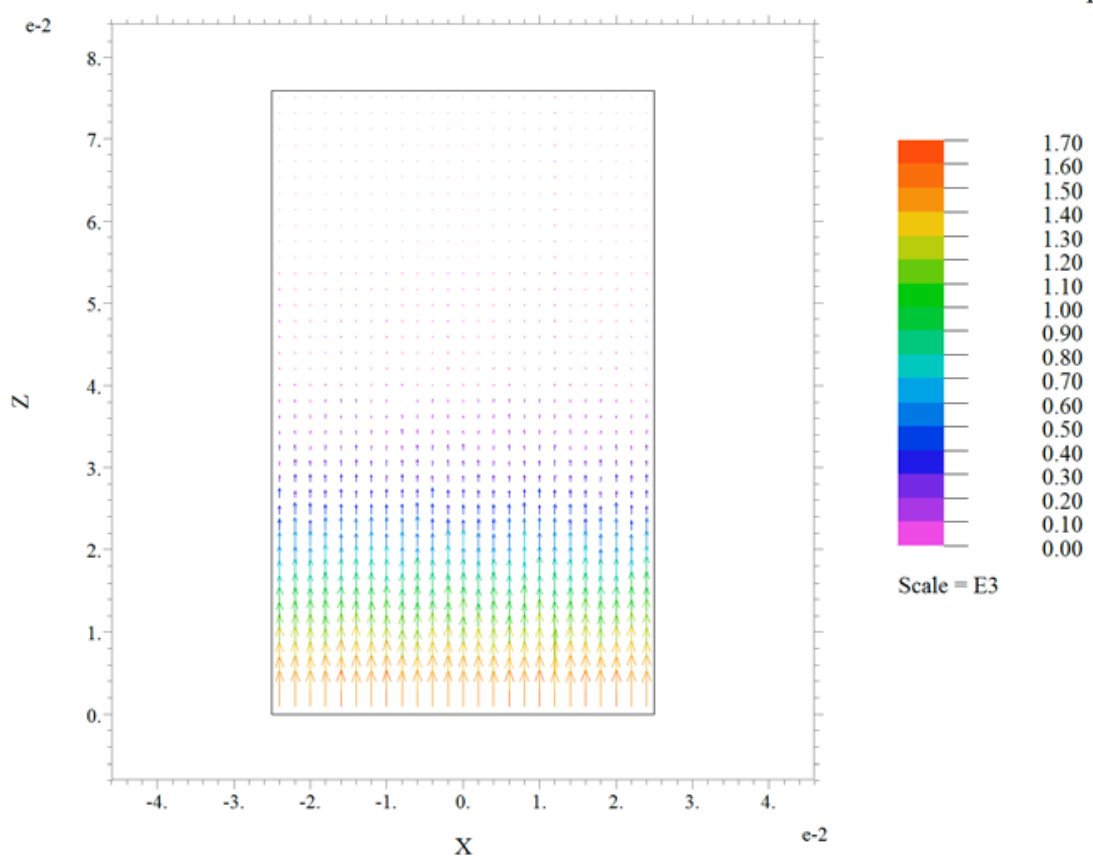

Fig. 18. The heat flux at $t=5^{*} 10^{3} \mathrm{~s}$ for $\lambda=0.5 \mathrm{~J} / \mathrm{ms} \mathrm{K}$ 


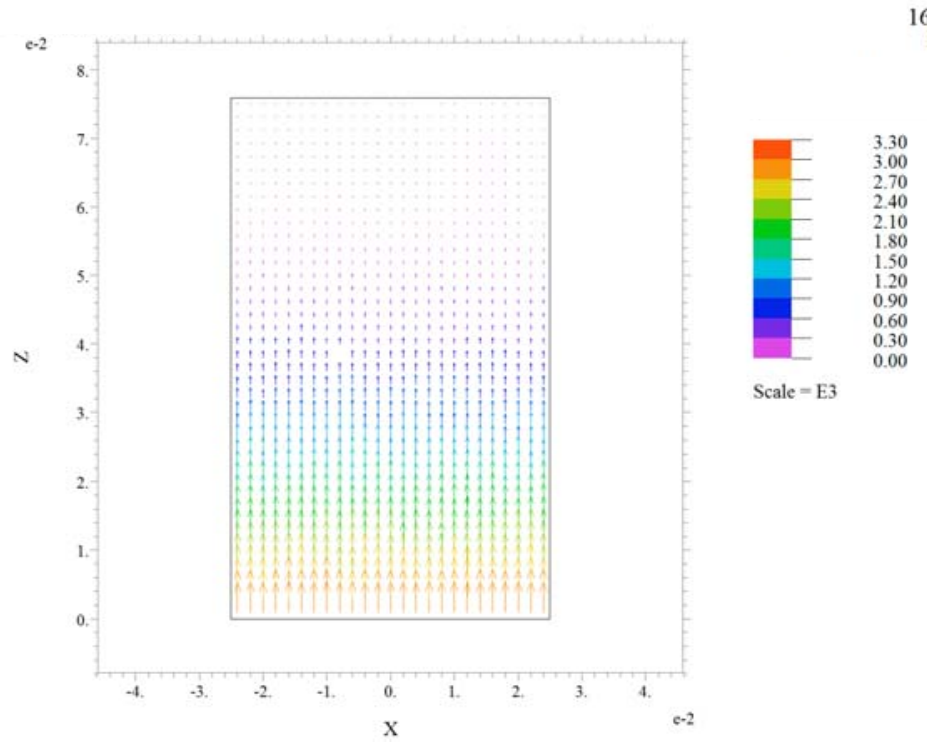

Fig. 19. The heat flux at time $t=5^{*} 10^{3} \mathrm{~s}$ for $\lambda=0.5 \mathrm{~J} / \mathrm{ms} \mathrm{K}$

The value of the coefficient of linear expansion of the soil influences the extent of the expansion effect after the temperature change on the bottom of the sample (Fig. 21), as expected, the coefficient of linear expansion does not influence the course of temperature changes (Fig. 20).

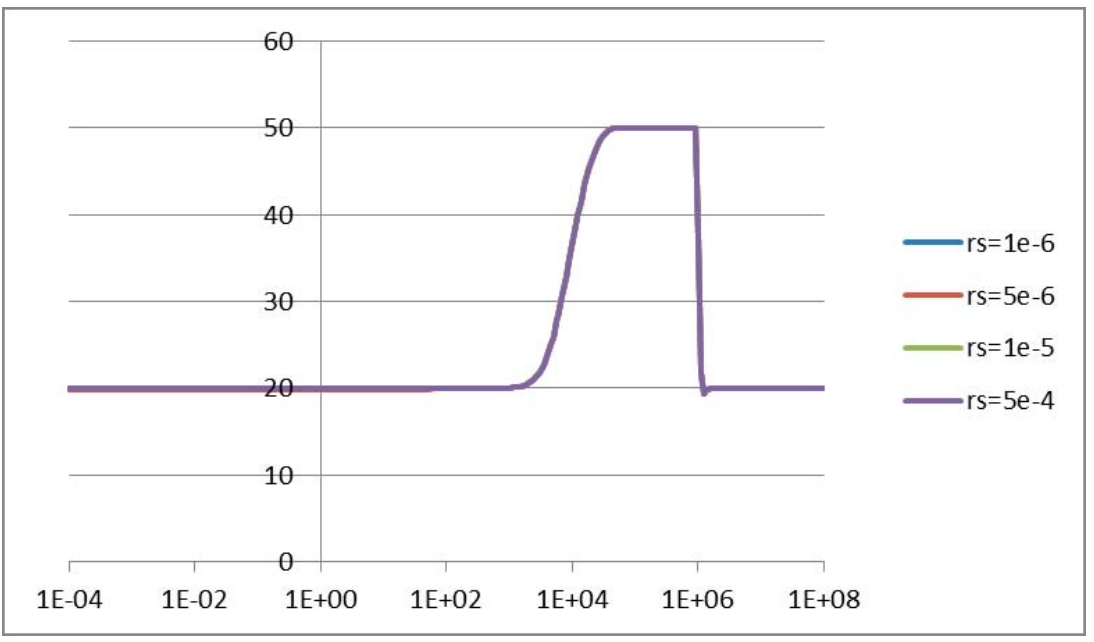

Fig. 20. The graph of temperatures in the center of the sample in time for different values of the coefficient of linear expansion of the sample 


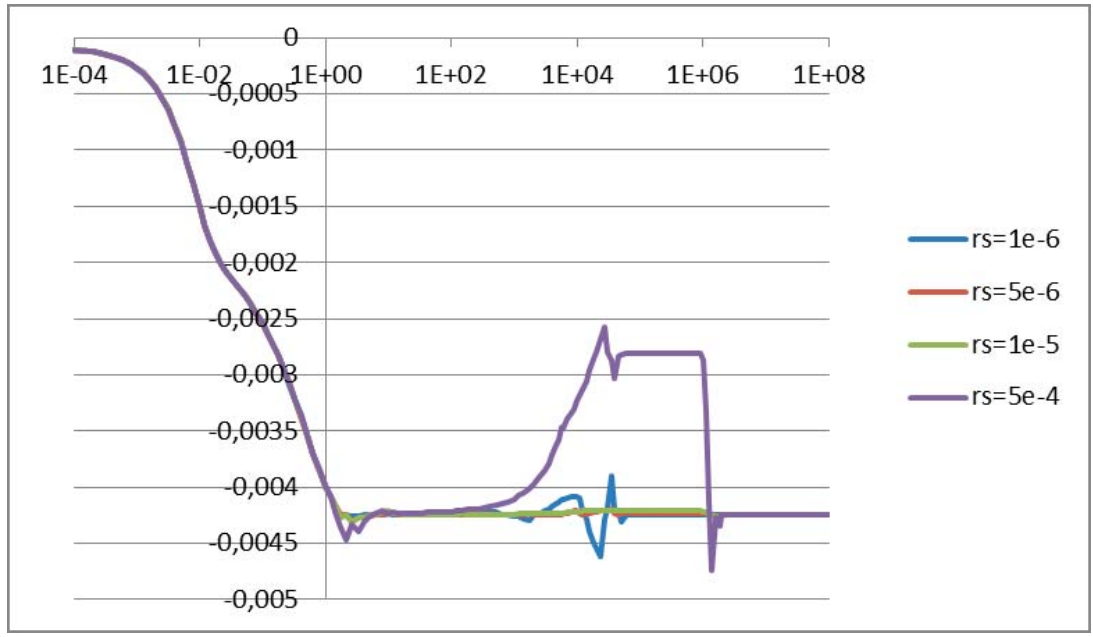

Fig. 21. displacement of the upper surface of the sample in time for different values of the coefficient of linear expansion of the sample

\section{SUMMARY AND CONCLUSIONS}

In this paper, the authors proposed to include in the process of consolidation of soil the effect of temperature on the evolution process of porous medium taking into account the changes in volume and shape viscosity of the skeleton and the viscous fluid flow under the influence of the temperature field. The mathematical model of thermal consolidation, presented in this work, taking into consideration rheological KelvinVoigt skeleton is a more accurate approximation of the real processes as the important aspect of it is lack of immediate displacement.

The experiment consisted in heating the sample from the bottom for a limited time after having reached an approximate steady state. The heating effect can be observed as an increase in the sample height and thus the relative displacement of its upper surface in the direction opposite to the force of gravity and the applied load. After stopping the heating, the sample height returned to values close to pre-heating. The effect of this expansion was more pronounced with the greater the value of the coefficient of linear expansion. Changes in the value of specific heat and thermal conductivity coefficient do not affect the final result of expansion but influence their speed. The graphs of displacement, stress distribution, filtration and heat flow field and temperature distribution, obtained directly from flex. V.6 PDE are consistent with both the objectives of the experiment and intuition, and show the correctness of the model.

The model can be used to solve problems considered important from the point of view of engineering practice. Especially taking into account that the theme of the stress-strain states in the rocks at greater depth requires a comprehensive analysis of 
geothermal processes and their influence on the behavior of rocks in the area of a significant temperature gradient. This may be of importance in connection with plans to build a mine at a depth much greater than today.

\section{REFERENCES}

[1] Auriaullt J.L., Dynamic behaviour of porous media, Transport Processes in Porous Media, Kluver Academic Publishers, 1991, 471-519.

[2] Auriault J.L., Sanchez Palencia E., Etude de comportement macroscopique d'un milieu poreux sature deformable, Journal de Mecanique, 1977b, 16(4), 575-603.

[3] Auriault J.L., Strzelecki T., Bauer J., He S., Porous deformable media by a very compressible Fluid, Eur. J. Mech. a/Solid, 1990, 9, 4, 373-392.

[4] Bartlewska-Urban M., Strzelecki T., One-dimensional consolidation of the porous medium with the rheological Kelvin-Voigt skeleton, Studia Geotechnica et Mechanica, 2008, Vol. 30, No. 1/2, $115-122$.

[5] BARTLEwSKa M., The doctoral dissertation on the theme: Określenie parametrów efektywnych modeli reologicznych gruntów spoistych, Politechnika Wrocławska, Faculty of Geoengineering, Mining and Geology, Wrocław, 2009.

[6] BARTLEWSKa M., STRZelecki T., Equations of Biots consolidation with Kelvin-Voight rheological frame, Studia Geotechnica et Mechanica, 2009, Vol. XXXI, No. 2, 3-15.

[7] Bartlewska M., STRZelecki T., One-dimensional consolidation of the porous medium with the Rheological Kelvin-Voight skeleton, Studia Geotechnica et Mechanica, 2008, Vol. XXX, No. 1-2.

[8] Bıт M.A., General theory of three-dimensional consolidation, J. Appl. Phys., 1941, No. 12, 155.

[9] Biот M.A., General Solutions of the Equations of Elasticity and Consolidation of a Porous Material, J. Appl. Mech., 1956, 23.

[10] Bensoussan A., Lions J.L., Papanicolau G., Asymptotic analysis for periodic structures, North Holland Publishing Company, Amsterdam, 1978.

[11] Coussy O., Mechanics of Porous Continua, John Wiley \& Sons, 1995.

[12] Coussy O., Mechanics and physics of porous solids, John Wiley \& Sons, 2011.

[13] Kisiel I., DERSKI W., IZBICKI R.J., MrÓz Z., Mechanika skat i gruntów, series Mechanika Techniczna, Vol. VII, PWN, Warszawa, 1982.

[14] KisIEL I., Reologiczne równania stanu ośrodków quasiliniowych, Polish Academy of Sciences, Wrocław Branch, Wrocław, 1980.

[15] Kowalski S.J., Musielak G., RYbicki A., Drying Processes in Context of the Theory of Fluid Saturated Porous Materials, J. Theoretical and Applied Mechanics, 1998, 36, 3.

[16] Kowalski S.J., Musielak G., RYbicki A., Drying Processes in Context of the Theory of Fluid Saturated Porous Materials, J. Theoretical and Applied Mechanics, 1998, 36, 3.

[17] Kowalski S.J., Musielak G., Drying Processes in Aspect of the Theory of the Fluid Saturated Porous Materials, Proceedings of the Fifth International Conference on Composities Engineering, ICCE/5, ed. D. Hui, 1998, 487-488.

[18] Kowalski S.J., MusielaK G., RYBICKi A., Theory of Drying Processes as an Aspect of Poromechanics, "Poromechanics - A Tribute to Maurice A. Biot", ed. J.F. Thimus et al., 433-437, A.A. Balkema, Roterdam/Brookfield, 1998.

[19] KRÖNER E., Effective elastic moduli of periodic and random media: a unification, Mechanics Research Communication, 1980, 7(5), 323-327.

[20] ReINER M., Deformation, strain and flow, H. K. Lewis, London, 1960. 
[21] Rubinstein J., Torquato S., Flow and random porous media: mathematical formulation, variational principles and rigorous bounds, J. Fluid Mech., 1989, 206, 25-46.

[22] Sanchez-Palencia E., Non homogeneous Media and Vibration Theory, Lecture Notes in Phisics, 127, Springer-Verlag, Berlin, 1980.

[23] STRZELECKI T., Loi de comportement dans la theorie de la consolidation electrohydrodynamique, Stud. Geotech., 1979, Vol. 1, No. 3/4.

[24] STRZELECKI T., Model termokonsolidacji gruntów ilastych z uwzględnieniem procesów elektrokinetycznych, Współczesne problemy naukowo badawcze budownictwa lądowego i wodnego, Oficyna Wydawnicza Politechniki Wrocławskiej, Wrocław, 2007.

[25] Strzelecki T., Kostecki S., ŻAK S., Modelowanie przeplywów przez ośrodki porowate, Dolnośląskie Wydawnictwo Edukacyjne, 2008.

[26] Szefer G., Non-linear Problems of consolidations theory, Mat. III Kolokwium PolskoFrancuskiego, 22-24 kwietnia 1980.

[27] StrZelecki T., BARTLEWSKA-URBAn M., Numerical calculations of the consolidation of flotation waste landfill "Żelazny Most" based on Biot's model with the Kelvin-Voight rheological skeleton, Archives of Civil Engineering, Archives of Civil Engineering, 2011, Vol. 57, Iss. 2, 199-213.

[28] Flex PDE v.6: www.pdesolutions.com 